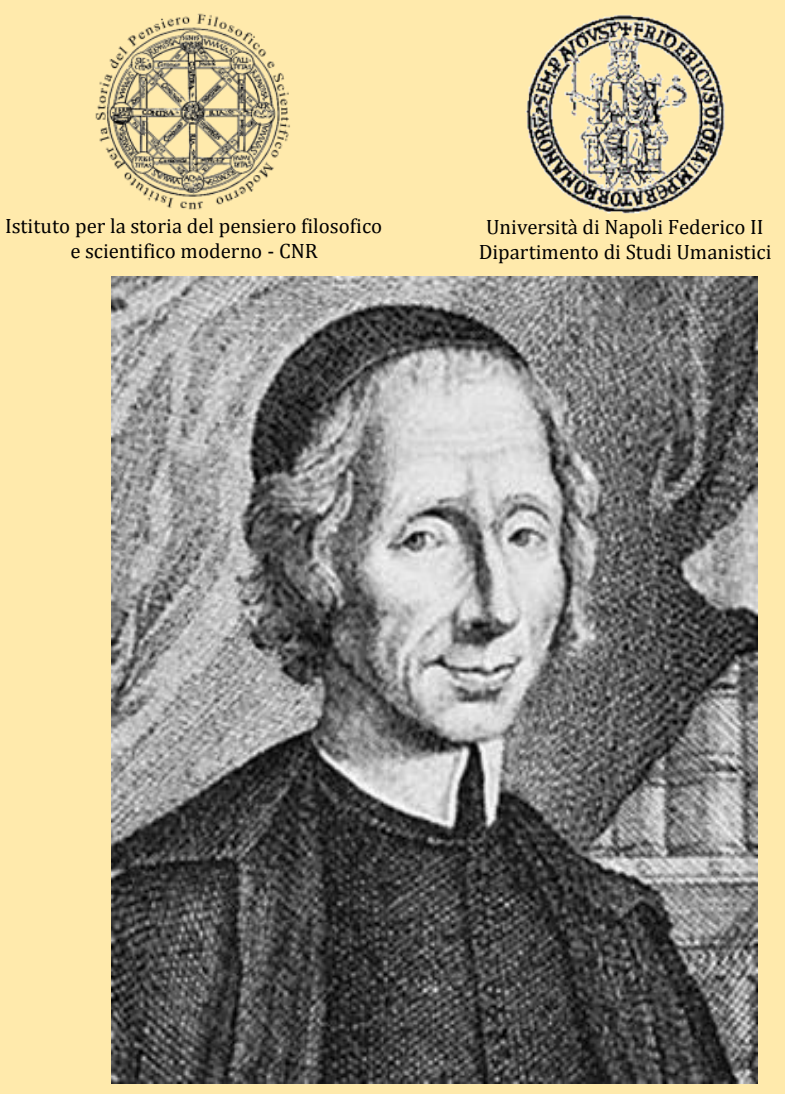

Napoli, Via Porta di Massa 1 - III piano Scala A 15-16 dicembre 2015

\section{Malebranche e i suoi contemporanei}

15 dicembre 2015

Ore 9.30

Giuseppe Cacciatore (Università di Napoli Federico II)

Raffaele Carbone (Università di Napoli Federico II / IHPC - UMR 5037 - Lyon)

Manuela Sanna (ISPF-CNR)

Accoglienza e introduzione ai lavori

1. Malebranche e i suoi interlocutori: debiti, critiche, polemiche e confronti

Presiede: Fulvio Tessitore

Ore 10.00

Raffaele Carbone (Università di Napoli Federico II / IHPC - UMR 5037 - Lyon)

Vere e false idee: la polemica tra Arnauld e Malebranche

Ore 10.30

Antonella Del Prete (Università della Tuscia)

Aperte polemiche e debiti nascosti: Pierre-Sylvain Régis e

Malebranche

Ore 11.00 - Discussione

Ore 11.30 - Pausa

Presiede: Giuseppe Cacciatore

Ore 11.45

Giuseppe D'Anna (Università di Foggia)

Spinoza e Malebranche a confronto

\section{Ore 12.15}

Francesco Piro (Università di Salerno)

L'argomento del miracolo perpetuo. Disposizioni naturali e leggi di natura nella critica leibniziana dell'occasionalismo

Ore 12.45 - Discussione

Ore 13.15 - Pausa pranzo
2. Malebranche e il malebranchismo: critiche e riprese

Presiede: Enrico Nuzzo

Ore 15.15

Roberto Evangelista (ISPF-CNR)

Idee e corpi. Locke critica la "visione in Dio"

Ore 15.45

Luisa Simonutti (ISPF-CNR)

Da Gerdil a Marugi: riprese malebranchiane e letture lockiane

Ore 16.15 - Discussione

Ore 16.45 - Pausa

\section{Malebranche e l'Italia nel XVIII secolo}

3.1. Malebranche e Vico

Presiede: Giambattista Gori

Ore 17.00

Geri Cerchiai (ISPF-CNR)

La «Malebrancicae doctrinae constantia» e il De antiquissima Italorum sapientia di Vico

\section{Ore 17.30}

Paolo Fabiani (Università di Firenze)

Alcune considerazioni sul concetto di memoria in Malebranche e Vico

Ore 18.00 - Discussione

\section{6 dicembre 2015}

3. Malebranche e I'Italia nel XVIII secolo

3.2. Malebranche nel contesto italiano ed europeo

Presiede: Cristina Santinelli

Ore 9.00

Enrico Nuzzo (Università di Salerno)

Ai limiti dell'ortodossia cristiana. Principio di semplicità e ordine naturale e storico in Malebranche, Vico, Feijoo

Ore 9.30

Pierre Girard (Université Jean Moulin Lyon III)

Diffusione e ricezione di Malebranche nel primo illuminismo napoletano

Ore 10.00

Alessandro Stile (ISPF-CNR)

Passioni di spada e passioni di croce

Ore 10.30 - Discussione

Ore 11.00 - Pausa

\section{Malebranche oltre il XVIII secolo}

Presiede: Luisa Simonutti

Ore 11.15

Cristina Santinelli (Università di Urbino Carlo Bo)

Nicolas Malebranche nelle Letture per la storia della filosofia moderna (1860) di Ausonio Franchi

\section{Ore 11.45}

Giambattista Gori (Università di Milano)

Malebranche e la ricerca della identità perduta. Fortune e incerti di una filosofia tra Ottocento e Novecento

Ore 12.15 - Discussione e conclusione dei lavori

Comitato scientifico-organizzativo: Giuseppe Cacciatore (Università di Napoli Federico II), Raffaele Carbone (Università di Napoli Federico II), Manuela Sanna (ISPF-CNR) 